\section{The effects of fasting during Ramadan on the concentration of serotonin, dopamine, brain- derived neurotrophic factor and nerve growth factor}

\author{
Abdolhossein Bastani, Sadegh Rajabi, \\ Fatemeh Kianimarkani \\ Biochemistry Department, Shahid \\ Beheshti University of Medical Sciences, \\ Tehran, Iran
}

\begin{abstract}
Neurotransmitters and neurotrophic factors are signaling molecules that play a crucial role in cell proliferation, differentiation, survival and functions of neurons. It is believed that caloric restriction could help the health of the nervous system by affecting the synthesis of neurotrophins and neurotransmitter and oxygen radical metabolism. The objective was to investigate the plasma levels of serotonin, dopamine, brain-derived neurotrophic factor (BDNF), and nerve growth factor (NGF) in 29 healthy fasted subjects ( 22 women and 7 men) during the month of fasting in Ramadan. The levels of these factors were measured (using ELISA method) three times, 2 days before the fasting month as a control, on the $14^{\text {th }}$ and $29^{\text {th }}$ day of Ramadan as test groups. In addition, these factors were investigated in the group of women only. According to our investigation, the plasma levels of serotonin, BDNF and NGF were significantly increased during fasting month of Ramadan. In detail, the levels of these factors were increased in $14^{\text {th }}$ and 29th day test groups compared to controls $(\mathrm{P}<0.05)$. Moreover, these levels were significantly increased on the $29^{\text {th }}$ day compared to the $14^{\text {th }}$ day test groups, but there were no differences between dopamine levels in all groups. Furthermore, the results obtained in women's groups were the same as those obtained in previous groups. Our findings suggest that plasma levels of serotonin, BDNF and NGF were significantly increased during fasting month of Ramadan.
\end{abstract}

\section{Introduction}

Neurons, in general, do not continue to divide after they differentiate and may have various damages and disorders in their structure and function during their life. ${ }^{1}$ It has been reported that the prevalence of neurological disease is increasing worldwide with the highest incidence and prevalence of these disorders in developing countries. Improving life style is one approach to prevent neuronal damage. ${ }^{2}$

Neurotrophic factors are biochemical molecules, which have an important role in differentiation and survival of neurons. These factors are known as neural growth factors, capable of repairing damaged neurons and thereby can prevent neurodegenerative disease. ${ }^{3}$ A number of studies have been conducted to verify the therapeutic features of these factors in maintaining and survival of the neurons in recent years. As a result, neurotrophic factors have been reported to have neuronal health benefits, making them appropriate drug candidates for treatment of a number of neurodegenerative diseases. However, remarkable hindrances remain and need to be solved before using these factors as therapeutic agents.4,5 Besides, recent studies have shown that neurotrophins have actions on other cells. For example, nerve growth factor (NGF) has an important role in the maintenance of a balanced interplay between the immune, endocrine and nervous systems. ${ }^{6}$

Previous studies on therapeutic features of these factors with cell cultures and animal models provided support for the hypothesis that neurotrophic factors could prevent neuronal death. Many of these studies have shown that neurotrophins could be considered as therapeutic agents. However first clinical trials led to variable results and severe side effects were observed. Dopamine and serotonin, as neurotransmitters, have neuroprotective effects. Dopamine has also been demonstrated to have neurotrophic effects. It is believed that low serotonin is one of the factors which are responsible for depressive symptoms in schizophrenic patients. 7,8 It has been reported that the consumption of tryptophan, as a precursor of serotonin, can lead to diminished depression. 9 Therefore, scientists have to find other ways to increase neurotrophic factors in human brain. A number of studies using animal models shows that calorie restriction or fasting, by increasing these factors, may have beneficial effects on brain function. ${ }^{10}$ One way to change life style for this purpose is the calorie restriction or fasting, which is one of the religious ceremonies in the month of Ramadan in Islamic countries. Ramadan is one of the five pillar of Islam observed by all healthy Muslim's of the world in an annual month of fasting when they abstain any form of drinking or eating during the day. ${ }^{11}$ The food deprivation during month long fasting in Ramadan has been the subject of extensive investigation including the effect on
Correspondence: Abdolhossein Bastani, Biochemistry Department, Shahid Beheshti University of Medical Sciences, 198396-3113 Tehran, Iran.

Tel : +98 2122439982 - Fax : +98 2122439784 E-mail: ab bastani2002@yahoo.com

Key words: Caloric restriction; Serotonin; Dopamine; BDNF; NGF.

Contributions: $\mathrm{AB}$ conceived, designed, and wrote the article; SR assisted in writing the manuscript; FK prformed the experimental work.

Conflict of interest: the authors declared no potential conlict of interest.

Received for publication: 11 January 2017.

Revision received: 24 April 2017.

Accepted for publication: 17 May 2017.

This work is licensed under a Creative Commons Attribution NonCommercial 4.0 License (CC BY-NC 4.0).

(C) Copyright A. Bastani et al., 2017

Licensee PAGEPress, Italy

Neurology International 2017; 9:7043

doi:10.4081/ni.2017.7043

patients with chronic liver disease, diabetes, cardiovascular disease, and cerebrovascular disease. ${ }^{12-14}$

Some of the studies showed an increased tissue concentrations of the amine and of its metabolite 5-hydroxyindoleacetic acid (5-HIAA) in some brain regions of the fasted rats. ${ }^{15}$ Naswood et al. reported that caloric restriction can alleviate the severity of neurochemical deficits and motor dysfunction in a Parkinson's disease model. They also showed that levels of glial cell line-derived neurotrophic factor, which has an important role in the survival of dopamine neurons, were increased significantly in the caudate nucleus of calorierestricted monkeys, suggesting a role for this factor in the anti-Parkinson's disease effect of the low-calorie diet. ${ }^{16}$

Duan et al. showed that the levels of brain-derived neurotrophic factor (BDNF) was significantly increased in the hippocampus, cerebral cortex, and striatum of rats maintained on a dietary restriction (DR) regimen compared to controls. 17 They reported that seizure-induced damage to hippocampal neurons could significantly reduced in rats maintained on DR, providing evidence for the role of diet in expression of neurotrophic factor and proving DR as an approach for reducing neuronal damage in neurodegenerative disorders. ${ }^{17}$ Seizure-induced damage to hippocampal neurons was significantly reduced in rats 
maintained on DR, providing evidence for the role of diet in expression of neurotrophic factor and proving DR as an approach for reducing neuronal damage in neurodegenerative disorders. ${ }^{17}$

The majority of studies in this field has been conducted on animals and there are a few or no studies which were done in human. Thus, to test the hypothesis that serum neurotrophines (BDNF, NGF) and neurotransmitters (dopamine and serotonin) increase after a period of fasting in human subjects, we conducted this study to investigate the changes of plasma levels of these neurotransmitters and neurotrophins in healthy fasted subjects during the month of fasting in Ramadan.

\section{Materials and Methods}

Twenty-nine healthy fasted subjects (22 women and 7 men; average age $38 \pm 3$ years) were included in the study. All of the subjects were healthy and there was no history of other diseases and medication intake. The consent forms that each subject had signed was obtained. Collection of blood samples was carried out during the month of fasting in Ramadan (three times, 2 days before the fasting month as a control group, $14^{\text {th }}$ and 29th day of Ramadan as test groups). Each time, about $20 \mathrm{~mL}$ of blood was collected from each person in tubes containing EDTA as anticoagulant. Samples were centrifuged immediately for $20 \mathrm{~min}$ utes at $2500 \mathrm{~g}$ at $4{ }^{\circ} \mathrm{C}$ and plasma was collected in $1.5 \mathrm{~mL}$ microtubes and stored at $80^{\circ} \mathrm{C}$ until they were analyzed. Plasma samples were assayed for BDNF, NGF, dopamine and serotonin levels. In addition, levels of these factors were also investigated in women only. This study was per- formed under the approval and permission of the Shahid Beheshti University of Medical Sciences and written informed consent that was obtained from all the volunteers and participants.

\section{Measurement of brain-derived} neurotrophic factor, nerve growth factor, dopamine, and serotinin in plasma samples

BDNF, NGF, dopamine, and serotinin measured using a commercially available direct enzyme-linked immunosorbent assay (ELISA) kit (DemedItec, Kiel, Germany) according to manufacturer's specifications. Both BDNF, NGF, dopamine, and serotinin concentration in plasma were calculated based on a standard curve. All samples were processed in one assay to minimize the interassay variability.

\section{Statistical analysis}

The data were analyzed using SPSS version 11 software (SPSS, Chicago, IL, USA). All data were analyzed by paired sample t-tests for group comparison and are expressed as mean \pm standard error of the mean, using $\mathrm{P}<0.05$ as the level of significance.

(n)
contents in the plasma of 100 subjects were

\section{Results}

Evaluation of the changes in plasma levels of brain-derived neurotrophic factor in fasted subjects during the month of Ramadan compared to the non-fasting state

Figure 1 shows the BDNF levels in plasma of the test, 14th (second) and 29th (third) day of Ramadan, and control groups. The BDNF levels in the second group were increased significantly by $25 \%$ compared to the control group $(\mathrm{P}<0.05)$. In addition, the levels of BDNF in the third group were significantly increased compared to second group by $29 \%$ and control group by $47 \%$. In our study, the levels of this factor were also investigated in women only. As shown in Table 1, there was a significant increase in the levels of BDNF in second $(295 \pm 4)$ and third $(417 \pm 7)$ groups compared to control $(221 \pm 3)$ and the third group as compared with the second group.

\section{Evaluation of the changes in plasma} levels of nerve growth factor in fasted subjects during the month of

Ramadan compared to the non-fasting state

The level of NGF in plasma of the test, 14th (second) and 29th (third) day of Ramadan, and control groups are shown in Figure 2.

Table 1. Effect of fasting on brain-derived neurotrophic factor levels in the group of women only. All data are expressed as means \pm standard error of mean; $\mathbf{P}<0.05$.

\begin{tabular}{lc} 
Groups & BDNF levels \\
Control (2 days before Ramadan) & $221 \pm 3$ \\
Second test (14th day of Ramadan) & $295 \pm 4$ \\
\hline Third test (14th day of Ramadan) & $417 \pm 7$ \\
\hline
\end{tabular}

BDNF, brain-derived neurotrophic factor.

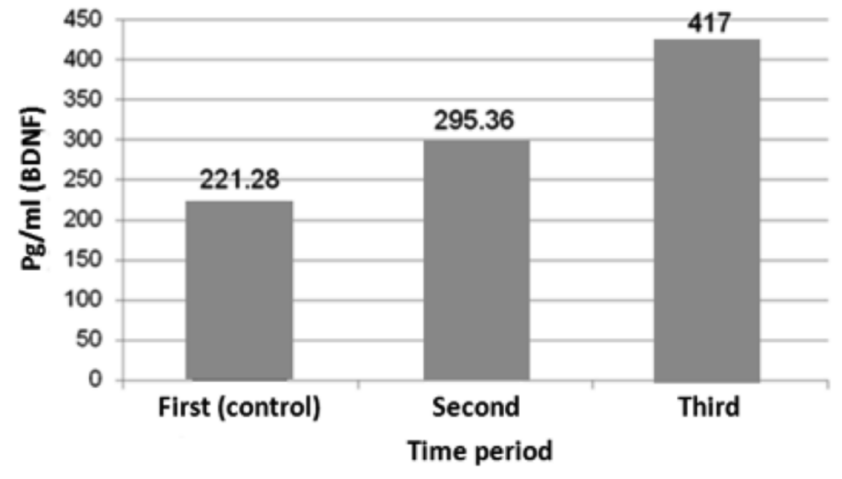

Figure 1. Effect of fasting on brain-derived neurotrophic factor levels on $1^{\text {th }}$ (second) and 29th (third) day of Ramadan compared to the control group. All data are expressed as mean \pm standard error of mean; $\mathrm{P}<0.05$.

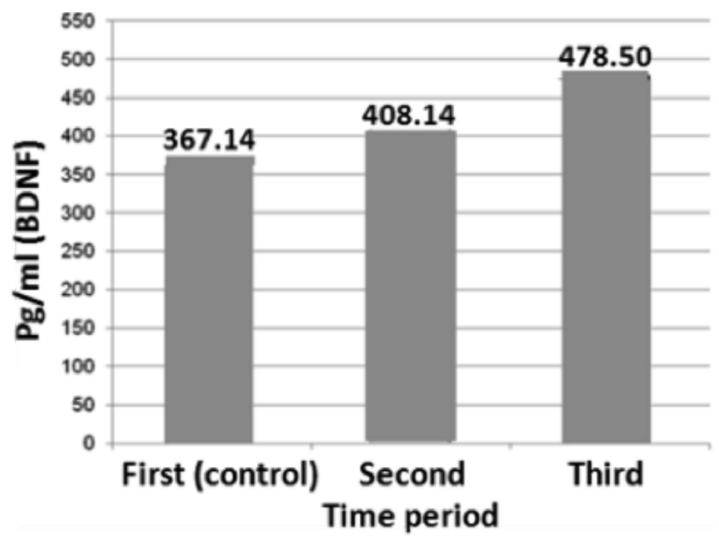

Figure 2. Effect of fasting on nerve growth factor levels on $14^{\text {th }}$ (second) and 29th (third) day of Ramadan compared to the control group. All data are expressed as mean \pm standard error of mean; $\mathrm{P}<\mathbf{0 . 0 5}$. 
NGF levels in the second group showed a significant increase by $10 \%$ compared to the control group $(\mathrm{P}<0.05)$. In addition, the levels of NGF in the third group were significantly increased compared to the second group by $14.7 \%$ and control group by $23.2 \%$. In our study, the levels of this factor were also investigated in women only. The level of NGF was significantly different between second $(376 \pm 5.2)$ and third groups $(479 \pm 11.6)$ compared to the control group $(367 \pm 11.2)$. We also found that the level of NGF between second and third groups were significantly different from each other (Table 2).

Evaluation of the changes in plasma levels of dopamine in fasted subjects during the month of Ramadan compared to the non-fasting state

The levels of dopamine in plasma of the test, 14th (second) and 29th (third) day of Ramadan, and control groups are shown in Figure 3. As shown in the figure, there was no significant difference between second group and control in dopamine levels $(\mathrm{P}<0.05)$. Accordingly, the levels of dopamine showed no significant differences between the third, second and control groups. As shown in Table 3, there were no significant between-group differences in dopamine levels in women only.

Evaluation of the changes in plasma levels of serotonin in fasted subjects during the month of Ramadan compared to the non-fasting state

Plasma levels of serotonin in the test, $14^{\text {th }}$ (second) and 29th (third) day of Ramadan, and control groups are shown in Figure 4. Plasma serotonin levels in the second group significantly increased by $33.4 \%$ when compared to the control group $(\mathrm{P}<0.05)$. In addition, the levels of serotonin in the third group were significantly increased compared to the second group by $14.7 \%$ and control group by $43.1 \%$. Furthermore, we conducted a study to investigate the levels of serotonin in women only. According to the Table 4, there were significant differences between the levels of serotonin in second $(57.4 \pm 1.4)$ and third (69.2 \pm 1.4$)$ groups compared to control (46.1 \pm 1.3$)$ and the third group as compared with the second group.

\section{Discussion}

All type of fastings, including fasting in Ramadan, has been the subject of intensive study by scientist and clinician. All tissues and organs, including brain, response in one way or another to caloric restriction. Although brain-derived neurotrophic factor and neurotransmitters play a significant role in mental health few or no studies has been conducted on human subject under caloric restriction.

There are reports indicating of a correlation between fasting and BDNF levels during fasting. One of the comprehensive studies addressing this correlation was carried out by Krisztina Marosi and his colleague. ${ }^{18}$ Their investigations showed that fasting/food deprivation can also induce BDNF expression in neuronal circuits involved in cognition by increasing their

Table 2. Effect of fasting on nerve growth factor levels in the group of women only. All data are expressed as means \pm standard error of mean; $\mathbf{P}<0.05$.

\begin{tabular}{lc} 
Groups & NGF levels \\
Control (2 days before Ramadan) & $367 \pm 11.2$ \\
Second test (14th day of Ramadan) & $376 \pm 5.2$ \\
\hline Third test (14th day of Ramadan) & $479 \pm 11.6$ \\
\hline
\end{tabular}

NGF, nerve growth factor.

Table 3. Effect of fasting on dopamine levels in the group of women only. All data are expressed as means \pm standard error of mean; $P<0.05$.

Groups Dopamine levels

\begin{tabular}{ll} 
Control (2 days before Ramadan) & $701 \pm 14.7$ \\
Second test (14th day of Ramadan) & $739 \pm 14.9$ \\
\hline Third test (14th day of Ramadan) & $759 \pm 16.4$ \\
\hline
\end{tabular}

Table 4. Effect of fasting on serotonin levels in the group of women only. All data are expressed as means \pm standard error of mean; $P<0.05$.

\begin{tabular}{lc} 
Groups & Serotonin levels \\
Control (2 days before Ramadan) & $46.1 \pm 1.3$ \\
Second test (14th day of Ramadan) & $57.4 \pm 1.4$ \\
\hline Third test (14th day of Ramadan) & $69.2 \pm 1.4$ \\
\hline
\end{tabular}

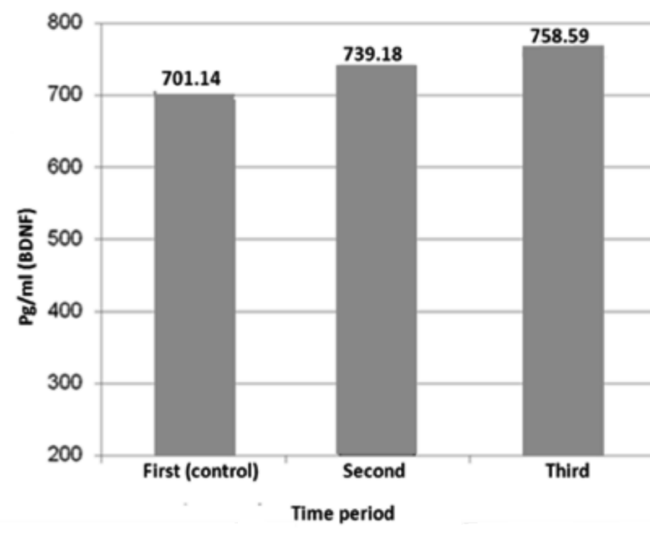

Figure 3. Effect of fasting on dopamine levels on $14^{\text {th }}$ (second) and 29th (third) day of Ramadan compared to the control group. All data are expressed as mean \pm standard error of mean; $P<0.05$.

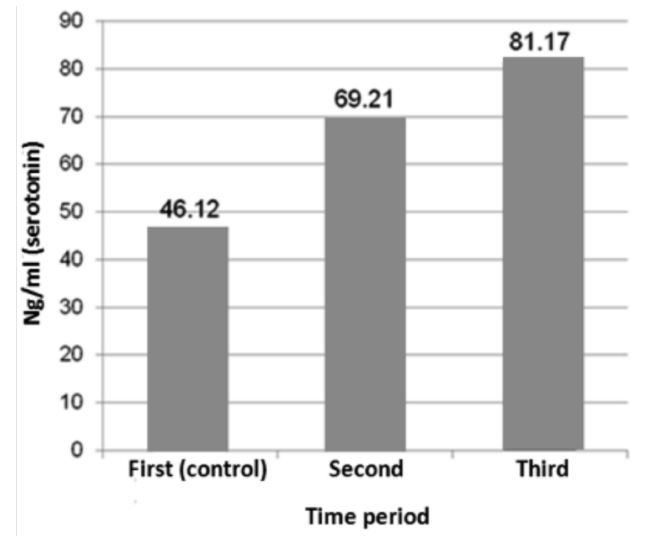

Figure 4. Effect of fasting on serotonin levels on $14^{\text {th }}$ (second) and $29^{\text {th }}$ (third) day of Ramadan compared to the control group. All data are expressed as mean \pm standard error of mean; $P<0.05$. 
activity, and by shifting cellular energy substrate utilization from glucose to ketones. They also added that intermittent fasting and exercise through BDNF induce the neurogenesis, by promoting the differentiation of neurons from stem cells, and the survival and synaptic integration of newly generated neurons. ${ }^{18}$ Alberto Del Arco et al. conducted a study to investigate the effect of longterm caloric restriction on neurotransmitters release and working memory performance. They showed that caloric restriction can cause to the elevation of BDNF in some region of the rat's brain. ${ }^{19}$ Araya et al. evaluated the effect of 3-month reduced-calorie diet (RCD) on serum BDNF concentrations in overweight and obese subjects. They reported that caloric restriction results in an increase in serum levels of BDNF in their studied subjects and therefore, they suggested that BDNF may be modulated in humans through diet composition. ${ }^{20}$ There also are several investigations, which indicate that the calorie restriction has beneficial effects on neurotrophic factors such as BDNF in the brain and that the elevation of this factor can improve the neurogeneration in the nervous system. $16,21-24$

In this study, we observed a significant increase in plasma BDNF levels in fasted subjects when compared to the non-fasting state as a control. We also studied the effect of fasting on nerve growth factor as an important factor in growth, differentiation and survival of target neurons in the nervous system. According to our findings, the levels of plasma NGF were significantly increased in fasted subjects when compared with control and this indicated that fasting may cause to improve brain health through the induction of the NGF levels.

There is, however, no previous evidence on the effect of fasting or caloric restriction on NGF levels. According to our results, the levels of serotonin in plasma of fasting states were significantly higher than those levels in non-fasting controls. Fuenmayorl and Garcia reported that fasting leads to increase the turnover of brain 5HT and suggested that the fasting state may increase 5-HT turnover in all brain areas. Moreover, they asserted that this increased tunover appears to be accompanied, in the hypothalamus, by an increased neuronal release of the amine. 15 Japanese researchers have found that short-term fasting can lead to increase in extracellular 5-hydroxyindole-3-acetic acid (5-HIAA), and the serum concentration of tryptophan (a precursor amino acid of 5-HT) also increased in the fasting group. 25

Other studies also show that restricted feeding increases the levels of serotonin. Jeong Won Jahng, et al. reported that Five weeks of food restriction in rat model markedly increased serotonin levels in hypothalamus although, the serotonin turnover 5-HIAA/5-HT decreased.26 However, there are some studies, which have different results than those obtained in our investigation. For example, in a study conducted to investigate the changes in metabolism of serotonin and sex differences in changes of this neurotransmitter in 4 weeks food restricted rats, it was found that both tryptophan (a precursor of serotonin) and serotonin levels markedly decreased. Meanwhile, after 4 weeks food restriction, the body weight of rats $20-25 \%$ were decreased.27 It appears that the difference between the results of the latter study and the present study is due to the type of dietary restrictions. It is believed that the long-term food restrictions can lead to depletion of tryptophan reservoirs and this may cause serotonin levels to decrease. While, in the present study, because of the sufficient and nutritious food, the levels of tryptophan were not decreased. In addition, in the study mentioned above, the body weight of feeding restricted rats was significantly lower than controls and this indicates that food was too restricted. In this study, no significant differences were observed in the plasma levels of dopamine in fasted subjects when compared with the control group. Rosberry AG, showed that food restriction or fasting could change the properties of somatodendritic dopamine release, and this may occur by increasing dopamine release. ${ }^{28}$ A recent review of impacts of fasting on mood studies reported that modified fasting in humans during the first 7 days increases the plasma levels of dopamine and some other neurotransmitters. ${ }^{29}$ However, Ishida, et al. showed that short-term fasting could affect on monoamine metabolism and thereby decreases the levels of extracellular striatal dopamine in rat pups. 25

Our results, in the case of dopamine levels, are inconsistent with the above mentioned findings. Probably the differences between the results of our study and previous reports arise from the different types of samples and dietary restriction methods which were used. According to the results of some investigations, serotonin can decrease the firing rate of dopamine secreting neurons and may lead to depression of dopaminergic neurons. In addition, the increase in serotonin release can also locally depress dopamine release. ${ }^{30-34}$ It appears that the increase in serotonin level in the present study partially depressed the dopamine release and it may be a reasonable explanation for no significant changes in dopamine levels in our study.

\section{Conclusions}

In conclusion, our findings suggest that plasma levels of serotonin, BDNF and NGF were significantly increased during fasting month of Ramadan. However, there is still much that needs to be investigated to better understand the underlying mechanisms.

\section{References}

1. Purves D, Augustine GJ, Fitzpatrick D, et al. Neuroscience IIrd edition. Generation of neurons in the adult brain. Sunderland: Sinauer Associates, Inc.; 2001.

2. Panksepp J. The neurochemistry of behavior. Ann Rev Psychol 1986;37:77107.

3. Garcia N, Santafe M, Tomas M, et al. Involvement of brain $\square$ derived neurotrophic factor (BDNF) in the functional elimination of synaptic contacts at polyinnervated neuromuscular synapses during development. J Neurosci Res 2010;88:1406-19.

4. Akagi M, Matsui $\mathrm{N}$, Akae $\mathrm{H}$, et al. Nonpeptide neurotrophic agents useful in the treatment of neurodegenerative diseases such as Alzheimer's disease. J Pharmacol Sci 2015;127:155-63.

5. Weissmiller AM, Wu C. Current advances in using neurotrophic factors to treat neurodegenerative disorders. Transl Neurodegener 2012;1:14.

6. Manni L, Albanesi M, Guaragna M, et al. Neurotrophins and acupuncture. Auton Neurosci 2010;157:9-17.

7. Allen GF, Ullah Y, Hargreaves IP, et al. Dopamine but not L-dopa stimulates neural glutathione metabolism. Potential implications for Parkinson's and other dopamine deficiency states. Neurochem Int 2013;62:684-94.

8. Peitl V, Vidrih B, Karlović Z, et al. Platelet serotonin concentration and depressive symptoms in patients with schizophrenia. Psychiatry Res 2016;239:105-10.

9. Rao TS, Asha M, Ramesh B, Rao KJ. Understanding nutrition, depression and mental illnesses. Indian $\mathrm{J}$ Psychiatr 2008;50:77.

10. Angelova A, Angelov B, Drechsler M, Lesieur S. Neurotrophin delivery using nanotechnology. Drug Discov Today 2013;18:1263-71.

11. Mohamed SY, Emara MH, Hussien HI, Elsadek HM. Changes in portal blood flow and liver functions in cirrhotics during Ramadan fasting in the summer; 
a pilot study. Gastroenterol Hepatol Bed Bench 2016;9:180-8.

12. Turin TC, Ahmed S, Shommu NS, et al. Ramadan fasting is not usually associated with the risk of cardiovascular events: a systematic review and metaanalysis. J Family Community Med 2016;23:73-81.

13. Alabbood MH, Ho KW, Simons MR. The effect of Ramadan fasting on glycaemic control in insulin dependent diabetic patients: a literature review. Diabetes Metab Syndr 2016;1871-4021.

14. Mehrpour M, Akhoundi FH, Rezaei Z. Effects of fasting during Ramadan on cerebrovascular hemodynamics: A transcranial Doppler study. Iran J Neurol 2016;15:23-7.

15. Fuenmayor LD, Garcia S. The effect of fasting on $5 \square$ hydroxytryptamine metabolism in brain regions of the albino rat. Brit J Pharmacol 1984;83:35762.

16. Maswood N, Young J, Tilmont E, et al. Caloric restriction increases neurotrophic factor levels and attenuates neurochemical and behavioral deficits in a primate model of Parkinson's disease. P Natl Acad Sci USA 2004;101:18171-6.

17. Duan W, Lee J, Guo Z, Mattson MP. Dietary restriction stimulates BDNF production in the brain and thereby protects neurons against excitotoxic injury. J Mol Neurosci 2001;16:1-12.

18. Marosi K, Mattson MP. BDNF mediates adaptive brain and body responses to energetic challenges. Trends Endocrinol Metab 2014;25:89-98.

19. Del Arco A, Segovia G, de Blas M, et al. Prefrontal cortex, caloric restriction and stress during aging: studies on dopamine and acetylcholine release,
BDNF and working memory. Behav Brain Res 2011;216:136-45.

20. Araya AV, Orellana X, Espinoza J. Evaluation of the effect of caloric restriction on serum BDNF in overweight and obese subjects: preliminary evidences. Endocrine 2008;33:300-4.

21. Andrade JP, Mesquita R, Assunção M, Pereira PA. Effects of food restriction on synthesis and expression of brainderived neurotrophic factor and tyrosine kinase B in dentate gyrus granule cells of adult rats. Neurosci Lett 2006;399: 135-40.

22. Duan W, Guo Z, Jiang H, et al. Dietary restriction normalizes glucose metabolism and BDNF levels, slows disease progression, and increases survival in huntingtin mutant mice. P Natl Acad Sci USA 2003;100:2911-6.

23. Lee J, Duan W, Long JM, et al. Dietary restriction increases the number of newly generated neural cells, and induces BDNF expression, in the dentate gyrus of rats. J Mol Neurosci 2000;15:99-108.

24. Lee J, Duan W, Mattson MP. Evidence that brain $\square$ derived neurotrophic factor is required for basal neurogenesis and mediates, in part, the enhancement of neurogenesis by dietary restriction in the hippocampus of adult mice. J Neurochem 2002;82:1367-75.

25. Ishida A, Nakajima W, Takada G. Shortterm fasting alters neonatal rat striatal dopamine levels and serotonin metabolism: an in vivo microdialysis study. Brain Res Dev Brain Res 1997;104: 131-6.

26. Jahng JW, Kim JG, Kim HJ, et al. Chronic food restriction in young rats results in depression-and anxiety-like behaviors with decreased expression of serotonin reuptake transporter. Brain Res 2007;1150:100-7.

27. Haider S, Haleem DJ. Decreases of brain serotonin following a food restriction schedule of 4 weeks in male and female rats. Med Sci Monit 2000;6:1061-7.

28. Roseberry AG. Acute fasting increases somatodendritic dopamine release in the ventral tegmental area. J Neurophysiol 2015;114:1072-82.

29. Fond G, Macgregor A, Leboyer M, Michalsen A. Fasting in mood disorders: neurobiology and effectiveness. A review of the literature. Psychiatry Res 2013;209:253-8.

30. Collingridge GL, Davies J. The influence of striatal stimulation and putative neurotransmitters on identified neurones in the rat substantia nigra. Brain Res 1981;212:345-59.

31. Dray A, Gonye T, Oakley N, Tanner T. Evidence for the existence of a raphe projection to the substantia nigra in rat. Brain Res 1976;113:45-57.

32. Ennis C, Kemp JD, Cox B. Characterisation of Inhibitory 5Hydroxytryptamine Receptors That Modulate Dopamine Release in the Striatum. J Neurochem 1981;36:151520.

33. Fibiger H, Miller J. An anatomical and electrophysiological investigation of the serotonergic projection from the dorsal raphe nucleus to the substantia nigra in the rat. Neuroscience 1977;2:975-87.

34. Gazzara RA, Takeda H, Cho AK, Howard SG. Inhibition of dopamine release by methylenedioxymethamphetamine is mediated by serotonin. Eur J Pharmacol 1989;168:209-17. 